\title{
Covariance analysis for total neutron cross sections based on a microscopic optical model potential
}

\author{
Ruirui $\mathrm{Xu}^{*}$, Zhigang Ge, Tingjin Liu, Yue Zhang, Xi Tao, and Zhi Zhang \\ China Nuclear Data Center, China Institute of Atomic Energy, P.O. Box 275(41), Beijing 102413, P.R. China
}

Received: 7 December 2017 / Received in final form: 1 March 2018 / Accepted: 28 May 2018

\begin{abstract}
The deterministic simple least square (LS) approach is employed in the covariance analysis of the total neutron cross section (n,tot) calculated by a microscopic optical potential, CTOM, which is based on a fundamental theory - Dirac Brueckner Hartree Fock. The sensitivity to the CTOM parameters is firstly systematically calculated for 77 stable nuclei in the range ${ }^{12} \mathrm{C}-{ }^{208} \mathrm{~Pb}$ within neutron energy $5-200 \mathrm{MeV}$. Then, an equivalent covariance of experimental data (EVexp) is constructed to describe the experimental data uncertainties and the systematic difference between experimental data and CTOM calculation. The variance and covariance of EVexp matrix are both evaluated via the Gaussian analysis to the ratios of measured (n,tot) cross sections and the CTOM calculations. In addition, a technique named "selection of effective points (SEP)" is suggested additionally to reduce the influence of the Peelle's Pertinent Puzzle problem in this work.
\end{abstract}

\section{Introduction}

As well known, covariance of cross sections make a big impact on the target accuracy design of modern nuclear facilities such as the reactors of Generation-IV [1]. Because experimental data on stable or unstable nuclei can be lacking for modern nuclear engineering studies, the evaluation of these nuclear data must rely heavily on theoretical calculations. Therefore, how to derive cross sections and associated covariance out of theoretical results is an attractive issue in the study of nuclear data.

The research based on a fundamental theory with better background and less freedom is believed to provide more confidence in theoretical prediction. Recently, a microscopic optical potential, CTOM, based on the Dirac Brueckner Hartree Fock (DBHF) theory has been developed in China Nuclear Data Center and Tuebingen University to globally describe the nucleon scattering from ${ }^{12} \mathrm{C}-{ }^{208} \mathrm{~Pb}$ for nuclear energy in the range $5-200 \mathrm{MeV}$ $[2,3]$. It is interesting to study the covariance of the CTOM predictions. Since CTOM is built on a microscopic theory, the covariance estimation should show some different features comparing to the traditional evaluation based on phenomenology. A very preliminary trial has been performed in reference [4] according to the uncertainty of the differential cross sections of nucleon scattering from ${ }^{40} \mathrm{Ca}$ and ${ }^{208} \mathrm{~Pb}$, however, due to its complexity, the

\footnotetext{
* e-mail: xrr-001@163.com
}

covariance of the CTOM prediction still deserve to be further studied, namely for its impact on other physics observable. Therefore, the purpose of this article is to further explore the covariance according to another important observable, the cross sections of (n,tot), and discuss more performance of CTOM predictions.

The methodologies of covariance evaluation can be simply classified as the deterministic least square (LS) approach and the stochastic Monte Carlo approaches [5]. Both have been used in the real nuclear data evaluation. Considering the merits of LS approach, such as involving more details of the experimental data and less time consuming, it is adopted in this work to analyze the covariance obtained from CTOM predictions. At the same time, some special methods related to the parameter sensitivity, the experimental covariance, and the Peelle's Pertinent Puzzle (PPP) effect are discussed in LS according to the properties of microscopic CTOM prediction.

The scheme of CTOM and its predictions have been presented in reference [3], in this paper, we focus on introducing the whole covariance evaluation process. The content is organized as follows. Firstly, a general description is presented to describe the LS approach in Section 2. Then, the sensitivity with respect of the CTOM parameters [2] are discussed in Section 3. The calculated total neutron cross sections of 77 stable nuclei in the region ${ }^{12} \mathrm{C}-{ }^{208} \mathrm{~Pb}$ are contained in this process, and a special parametrization for the uncertain components is designed to obtain the sensitivity. Thirdly, a so-called equivalent covariance of experimental data (EVexp) is introduced to describe the covariance based on the microscopic predictions and 


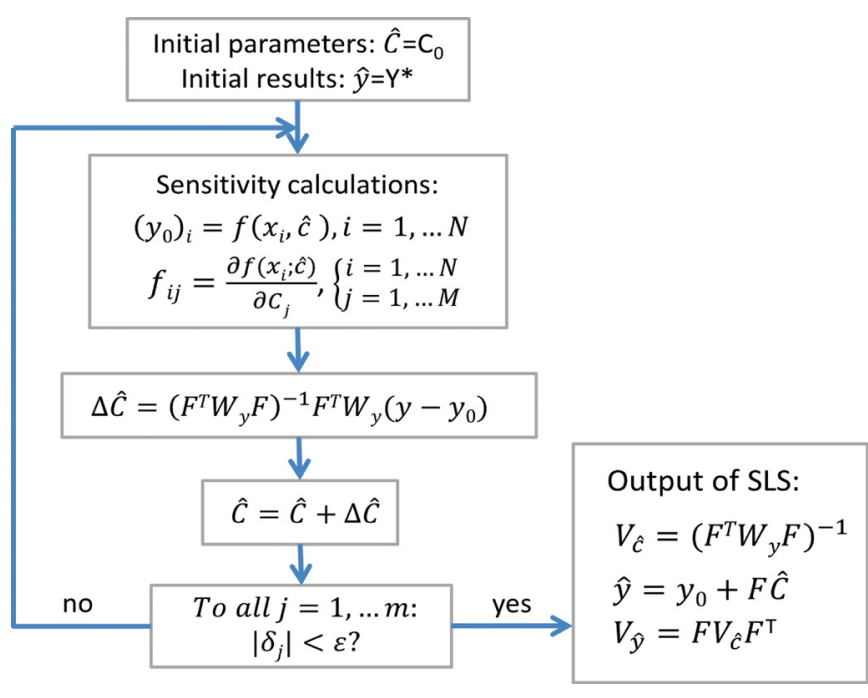

Fig. 1. The flow chart of conventional LS approach.

experimental data, which are illustrated in Section 4. In order to reduce the effect of PPP problem, we adopt a technique named "selection of effective points (SEP)" in LS calculation and the satisfactory result is presented in Sections 5 and 6. At last, this paper is summarized in Section 7.

\section{The deterministic simple LS approach}

Up to now, the LS approach is still very popular in the nuclear data and covariance evaluation. The conventional flow chart can be exhibited as Figure 1, and its full formalism can be referred in [6]. It is worthwhile to note that when assuming the prior probability of parameters of function $f$ as 1 , the LS approach can be expressed as the simple LS. It starts from an initial input of parameter array $\mathrm{C}_{0}$ and the initial derived values $\mathrm{Y}^{*}$ by the function $f$. The relevant parameter sensitivity matrix $\mathrm{F}$ is calculated for every variable $x_{i}$ by the function $f$. After that, the parameter array $\mathrm{C}_{0}$ is replaced by the new modified $\hat{C}$ via the uncertainty propagation from the experimental covariance $\mathrm{W}_{y}$ and theoretical sensitivities $F$. The process above will be iterated until the conditions set in the left bottom in Figure 1 are satisfied. The obtained $\hat{C}$ is believed to be the best estimate to fit the experimental data $y$ and the parameters covariance matrix $\mathrm{V}_{\hat{C}}$, the derived value $\hat{y}$ and the associated covariance $\mathrm{V}_{\hat{y}}$ are obtained as shown in Figure 1. In order to make use of the LS approach in this work, several issues related to CTOM are specifically discussed in the following.

\section{The sensitivity analysis}

The sensitivity of model parameter $F$ is one of the important components in LS, as mentioned in Section 2. It can exhibit the impact of uncertainties of the model parameter on the target function $f$, which is given by,

$$
F=\frac{\partial f}{\partial c}
$$

Normally, $F$ can be calculated through the analytical or numerical solution according to the target function $f$. However, the target function in CTOM is based on the microscopic theory but not phenomenological expression with a lot of adjusted parameters, which make it a difficulty to derive the $\mathrm{F}$ matrix in the conventional way. In order to solve this problem, we focus on finding the uncertain sources in the CTOM by performing a discussion throughout its whole formula scheme.

As presented in references [2,3], CTOM potential is built based on the relativistic self-energies by DBHF calculation, $\sum_{s}, \sum_{0}, \sum_{v}$, which represent the scalar part of self-energy, the time-like and space-like terms of the vector part, respectively. There is no free parameter in the solution procedure of nucleon self-energies within the nuclear matter. When the concentration is shifted to the finite nuclei, two uncertain parts are involved inevitably in the theoretical scheme of CTOM, namely the extrapolation of Dirac potentials $\mathrm{U}_{\mathrm{s}}, \mathrm{U}_{0}$ at lower density and the effective interaction range parameter $t$ in the improved local density approximation (ILDA). The sensitivity of $t$ can easily been calculated through incorporating small perturbation to the optimized $t$ values [3], but $\mathrm{U}_{\mathrm{s}}, \mathrm{U}_{0}$ at lower density region are different, because there are not direct parameter expression for them. In our previous trial in [4], the sensitivity is calculated by making perturbation on the auxiliary potentials points at $\rho=0.04 \mathrm{fm}^{-3}$ for the real parts and at $\rho=0.04$ and $0.06 \mathrm{fm}^{-3}$ for the imaginary parts, which are used in the Us and U0 extrapolation in the "full" density region for finite nuclei. In order to make more direct calculation, we introduce a simple quadratic curve $f 1$ as function of the nuclear matter density $\rho$ with the maximum of amplitude $F_{0}$ at $\rho=\rho_{0}$ while $\rho$ varies in [0, 0.08], $F_{0}$ indicates the amplitude of perturbation in sensitivity calculation,

where

$$
f 1(\rho)=a \rho^{2}+b \rho
$$

$$
a=\frac{-F_{0}}{\rho_{0}^{2}}, b=2 \frac{F_{0}}{\rho_{0}} .
$$

We define $f(\rho)=1+f 1(\rho)$, and the real and imaginary parts of Dirac potentials at lower density can be parameterized as

$$
U_{\text {new }}(\rho)=U_{\text {old }}(\rho) f(\rho)
$$

where $U_{\text {old }}$ stands for the original $\mathrm{U}_{\mathrm{s}}$ and $\mathrm{U}_{0}$ of CTOM. In this work, we select $\rho_{0}=0.04 \mathrm{fm}^{-3}$ and set $\left|F_{0}\right| \ll 1$ to keep $U_{\text {new }}$ as a tiny deviation from the $U_{\text {old }}$, which is required in the numerical solution of sensitivity. It is known that the step length is the important factor to derive the correct sensitivity in the numerical solution to equation (1). In this work, $F_{0}$ in equation (3) is adopted as 0.035 for all $U$ potentials, the $f, f 1$ and relevant perturbation to Dirac potentials are shown in Figures 2 and 3 . The absolute value of $F_{0}$ is set to 0.2 , in Figure 2 "minus" stands for $F_{0}=-0.2$ and "plus" for $F_{0}=0.2$. The corresponding change is propagated to the Dirac potentials as shown in Figure 2. The sensitivities are calculated based on this parameter 


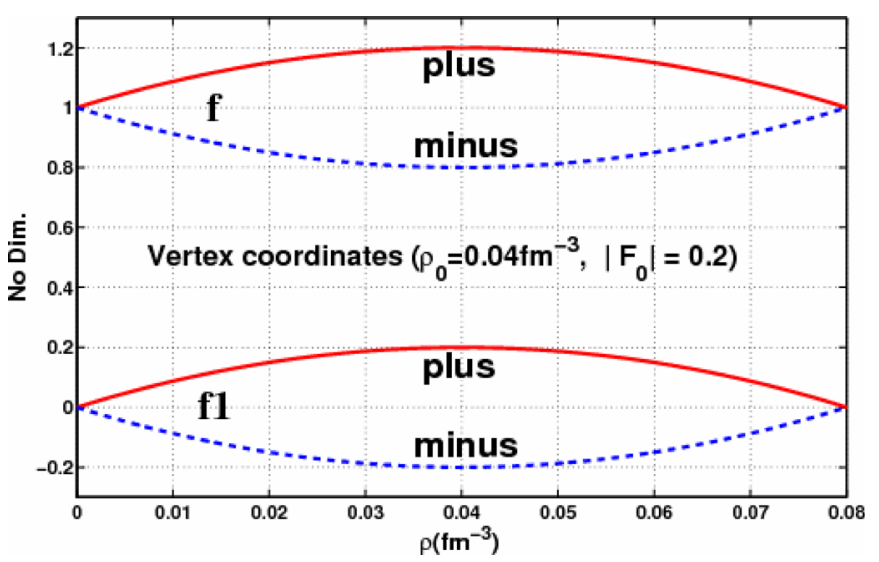

Fig. 2. The curves of perturbation function $f 1$ and $f$ at $\rho \leq 0.08 \mathrm{fm}^{-3}$.

variation. In addition, the variation step for the factor " $t$ " is taken as $\Delta t=0.01 t$ all calculations in ILDA as in references $[3,4]$.

As a result, six parameters in total are considered in describing the uncertainties in CTOM, they are t-Re, t-Im in ILDA and Real Us and U0 $\left(\operatorname{ReU}_{\mathrm{s}}, \mathrm{ReU}_{0}\right)$, Imaginary $\mathrm{U}_{\mathrm{s}}$, $\mathrm{U}_{0} \quad\left(\mathrm{ImUs}, \quad \mathrm{ImU}_{0}\right)$ respectively. Figure 4 shows the calculated sensitivities for the ${ }^{12} \mathrm{C}(\mathrm{n}$, tot) cross sections in $5 \leq \mathrm{E}_{n} \leq 200 \mathrm{MeV}$. One can observe that ${ }^{12} \mathrm{C}$ (n,tot) cross sections are sensitive to $\operatorname{ReU}_{\mathrm{s}}, \mathrm{ReU}_{0}, \mathrm{ImU}_{\mathrm{s}}$, t-Re, and the sensitivities of $\mathrm{ReU}_{\mathrm{s}}$ and $\mathrm{ReU}_{0}$ vary with energies more significantly.

In our calculation, the sensitivities have been derived for 77 stable nuclei in ${ }^{12} \mathrm{C}-{ }^{208} \mathrm{~Pb}$ in the incident energy region $5-200 \mathrm{MeV}$. The cross sections below $5 \mathrm{MeV}$ are not involved in this analysis due to the complex structures in (n,tot) cross section curves especially in the unresolved resonance region, whose structures can not be reproduced by optical model. We assemble the sensitivity results of ${ }^{12} \mathrm{C}-{ }^{208} \mathrm{~Pb}$ in one sensitivity matrix in our LS calculation. 77 nuclei in total are contained in this analysis, and they are ${ }^{12} \mathrm{C},{ }^{14} \mathrm{~N},{ }^{16} \mathrm{O},{ }^{19} \mathrm{~F},{ }^{20} \mathrm{Ne},{ }^{23} \mathrm{Na},{ }^{24} \mathrm{Mg},{ }^{27} \mathrm{Al},{ }^{28} \mathrm{Si},{ }^{31} \mathrm{P},{ }^{32} \mathrm{~S}$, ${ }^{35} \mathrm{Cl},{ }^{40} \mathrm{Ar},{ }^{39} \mathrm{~K},{ }^{40} \mathrm{Ca},{ }^{45} \mathrm{Sc},{ }^{48} \mathrm{Ti},{ }^{51} \mathrm{~V},{ }^{52} \mathrm{Cr},{ }^{55} \mathrm{Mn},{ }^{56} \mathrm{Fe},{ }^{59} \mathrm{Co}$, ${ }^{58} \mathrm{Ni},{ }^{63} \mathrm{Cu},{ }^{66} \mathrm{Zn},{ }^{69} \mathrm{Ga},{ }^{74} \mathrm{Ge},{ }^{75} \mathrm{As},{ }^{80} \mathrm{Se},{ }^{79} \mathrm{Br},{ }^{84} \mathrm{Kr},{ }^{85} \mathrm{Rb}$, ${ }^{88} \mathrm{Sr},{ }^{89} \mathrm{Y},{ }^{90} \mathrm{Zr},{ }^{93} \mathrm{Nb},{ }^{96} \mathrm{Mo},{ }^{99} \mathrm{Tc},{ }^{102} \mathrm{Ru},{ }^{103} \mathrm{Rh},{ }^{106} \mathrm{Pd}$, ${ }^{107} \mathrm{Ag},{ }^{112} \mathrm{Cd},{ }^{115} \mathrm{In},{ }^{120} \mathrm{Sn},{ }^{121} \mathrm{Sb},{ }^{128} \mathrm{Te},{ }^{127} \mathrm{I},{ }^{132} \mathrm{Xe},{ }^{133} \mathrm{Cs}$, ${ }^{138} \mathrm{Ba},{ }^{139} \mathrm{La},{ }^{140} \mathrm{Ce},{ }^{141} \mathrm{Pr},{ }^{144} \mathrm{Nd},{ }^{147} \mathrm{Pm},{ }^{152} \mathrm{Sm},{ }^{153} \mathrm{Eu}$, ${ }^{160} \mathrm{Gd},{ }^{159} \mathrm{~Tb},{ }^{164} \mathrm{Dy},{ }^{165} \mathrm{Ho},{ }^{166} \mathrm{Er},{ }^{169} \mathrm{Tm},{ }^{174} \mathrm{Yb},{ }^{175} \mathrm{Lu}$, ${ }^{180} \mathrm{Hf},{ }^{181} \mathrm{Ta},{ }^{184} \mathrm{~W},{ }^{187} \mathrm{Re},{ }^{192} \mathrm{Os},{ }^{193} \mathrm{Ir},{ }^{195} \mathrm{Pt},{ }^{197} \mathrm{Au},{ }^{202} \mathrm{Hg}$, ${ }^{205} \mathrm{Tl},{ }^{208} \mathrm{~Pb}$, respectively.

\section{The equivalent covariance of experimental data}

It is well known that the experimental data are important in LS to obtain the optimized parameters of the target function, and the experimental covariance matrix $\left(\mathrm{W}_{y}\right)$, as shown in Figure 1, also plays an important role to provide the weights of the each experimental data point in the fitting process. In this work, we introduce a so-called EVexp instead of the classical one to incorporate the experimental information in the covariance evaluation for
CTOM. EVexp is mainly constructed through exploring the systematic deviation between experimental data and the theoretical results, and the systematic evaluation of the total uncertainties of experimental data for ${ }^{12} \mathrm{C}-{ }^{208} \mathrm{~Pb}$. In total, more than 3000 sets of measurements for (n,tot) cross sections are involved, and the neutron incident energies En are ranging from $5-200 \mathrm{MeV}$.

In order to evaluate the EVexp matrix, we firstly define three uncertainties: ERR1, ERR2 and ERR3. In our estimation, ERR1 is the uncorrelated (statistical) uncertainty and comes directly from the reported total error of experimental data. As shown in Figure 5, we analyze all measured points in the 3000 sets of experimental data, each point is taken as one statistic sample in EVexp. A systematics function is obtained to evaluated the uncertainties for ${ }^{12} \mathrm{C}-{ }^{208} \mathrm{~Pb}$ in Figure 5. This uncertainty is taken as the statistic uncertainty ERR1.

In our correlated uncertainty estimation, the statistic Gaussian analysis is used to evaluate the ratio $=\mathrm{E} / \mathrm{C}$, namely the ratio of measurements "E" and theoretical results "C", to obtain the correlation. In order to get the better description to the $\mathrm{E} / \mathrm{C}$, we separate the analysis into two energy regions, 5-20 MeV and 20-200 MeV. Figure 6 shows the Gaussian analysis results for the $\mathrm{E} / \mathrm{C}$ in 20 $200 \mathrm{MeV}$. The FWHM of Gauss curve indicates the correlated uncertainty, ERR $2=0.026$ in $20-200 \mathrm{MeV}$, and the deviation between the Gauss curve center and 1.0 is the correlated uncertainty ERR3 $=0.038$ in 20 $200 \mathrm{MeV}$. In our work, ERR2 is adopted as the middlerange correlation in $20-200 \mathrm{MeV}$, and ERR3 is taken as the long-range correlation not only in $20-200 \mathrm{MeV}$ but also between $20-200 \mathrm{MeV}$ and 5-20 MeV, because it reflects the systematic deviation between measurements and theory predictions to some extend.

Based on the evaluation above, the matrix element $\rho_{i j}$ (correlation) in EVexp is built through the following formula,

$$
\left\{\begin{array}{l}
E R R 1^{2}+E R R 2^{3}+E R R 3^{2}, \quad(i=j) \\
E R R 2^{3}+E R R 3^{2}, \quad\left(i \neq j,\left(E_{i}, E_{j}\right) \in A^{2} \text { or } B^{2}\right) \\
E R R 3^{2}, \quad\left(i \neq j,\left(E_{i}, E_{j}\right) \in A \times B \text { or } B \times A\right)
\end{array}\right.
$$

where " $A$ " and " $B$ " stand for the energy regions of $5-20 \mathrm{MeV}$ and $20-200 \mathrm{MeV}$, respectively. It is noted that the coefficient of correlated uncertainties between different energies are preliminarily assumed as 1.0 in our calculation.

\section{The technique to reduce influence from Peelle's Pertinent Puzzle problem}

It is known that PPP problem is the inevitable issue when linear assumption is used to evaluate the nonlinear response function [7]. In order to reduce the PPP effect, we employ a method named SEP in our scheme. In SEP, the Gaussian analysis is also employed to pick out the proper points from the huge experimental database of the ${ }^{12} \mathrm{C}-{ }^{208} \mathrm{~Pb}$ (n,tot) cross sections. The adopted samples of experimental data used in LS are restricted to the data whose values are consistent with the theoretical prediction within 1 sigma. ${ }^{27} \mathrm{Al}(\mathrm{n}$, tot $)$ is chosen to illustrate the results 

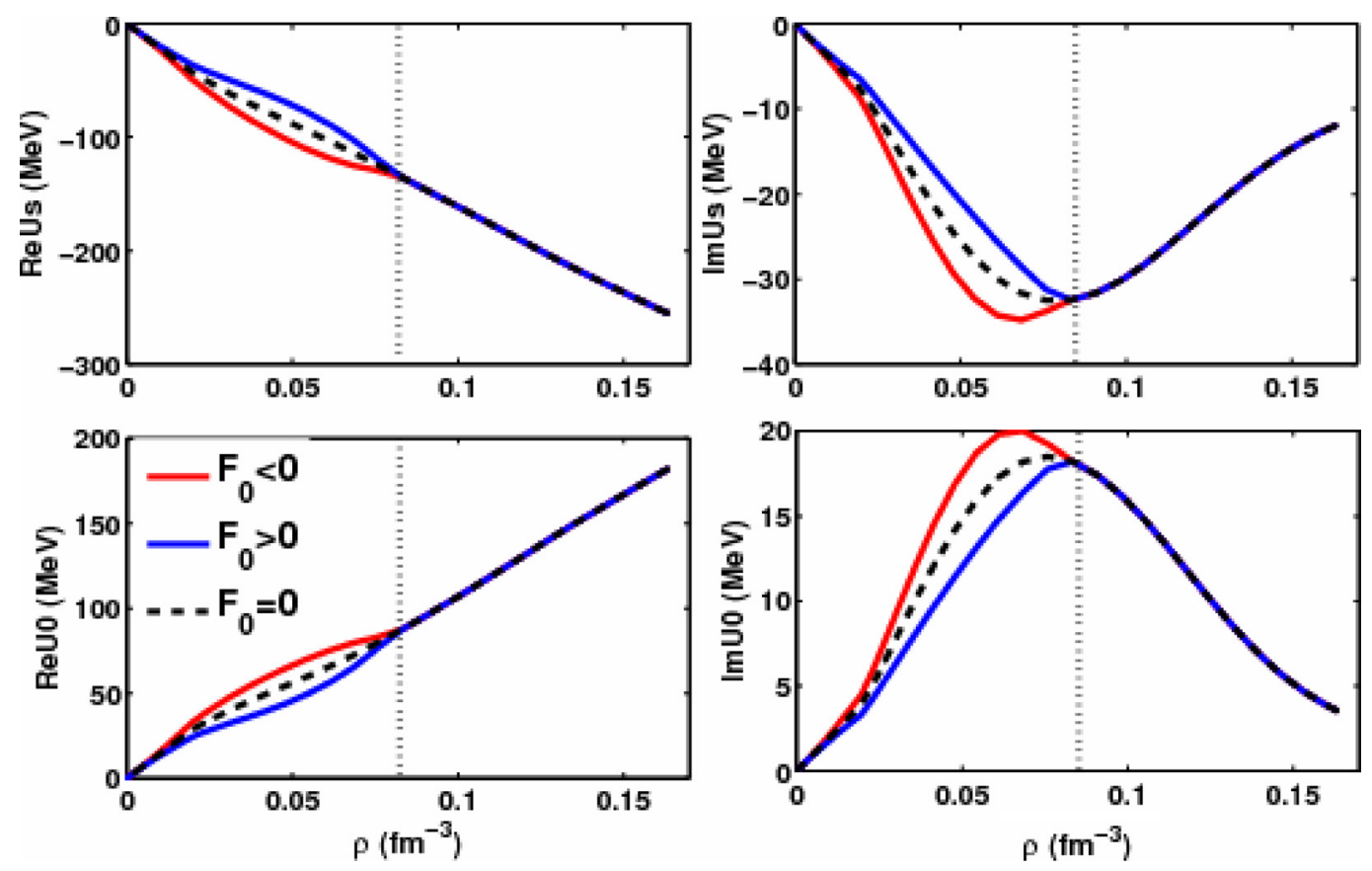

Fig. 3. The perturbation for $\mathrm{Us}_{\mathrm{s}}$ and $\mathrm{U}_{0}$ in this work.

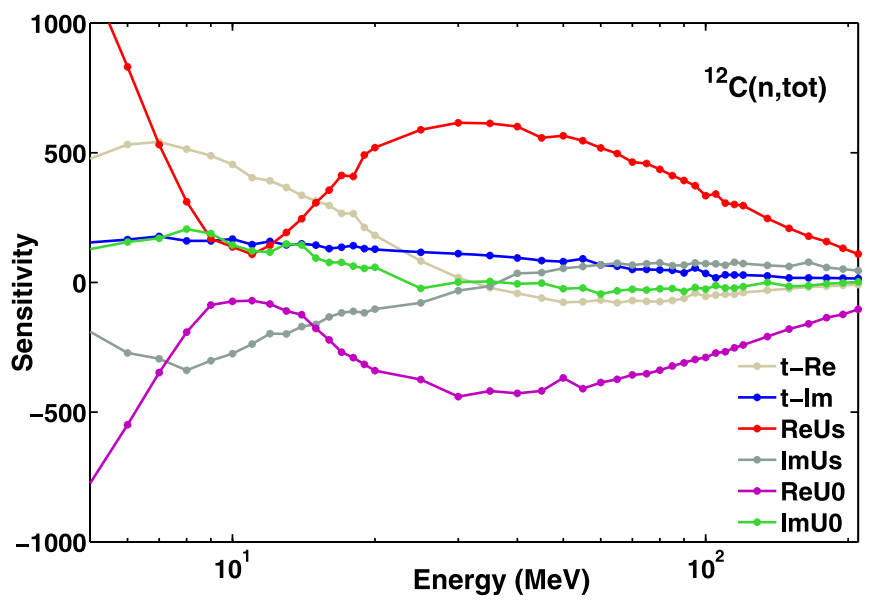

Fig. 4. The sensitivities of six parameters to the ${ }^{12} \mathrm{C}(\mathrm{n}$, tot $)$ cross sections.

of SEP in Figure 7. One can observe that the data derived from LS with SEP (red curve) is obviously improved and closer to the CTOM results (blue curve) and remains consistent with the measurements, while the one without SEP (black curve) is rather apart from others.

\section{The derived covariance for prediction of CTOM}

After combining the experimental and theoretical uncertainties using the sensitivity F, EVexp and SEP approach in LS, we derive the covariance of CTOM predictions. As an example, the ${ }^{27} \mathrm{Al}(\mathrm{n}$,tot) cross sections with derived uncertainties are shown in Figure 7 and the covariance

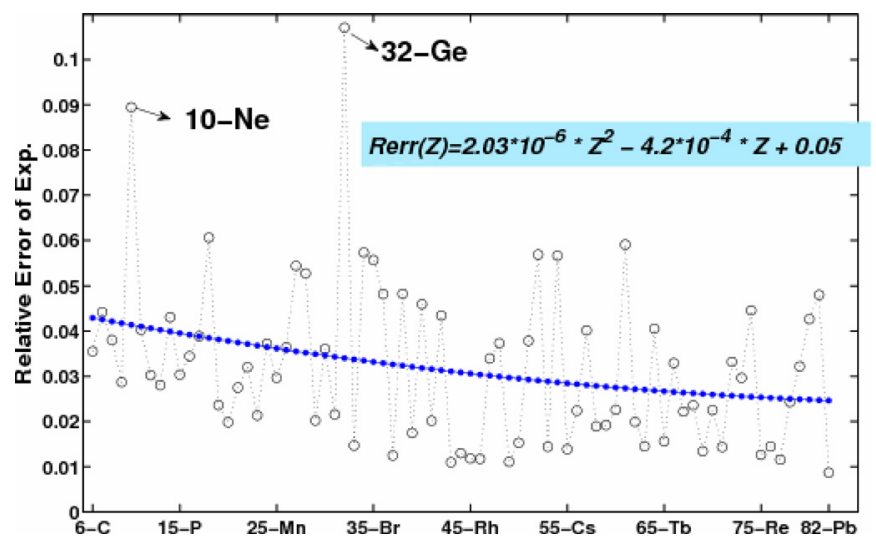

Fig. 5. The systematics study of the measured uncertainties of ${ }^{12} \mathrm{C}-{ }^{208} \mathrm{~Pb}$ (n,tot) cross sections.

(correlation) is shown in Figure 8. Labels of $\mathrm{X}$-axis and $\mathrm{Y}$ axis in Figure 8 are both energy mesh-grids from 5$200 \mathrm{MeV}$. In this work, the reduced $\chi^{2}$ is defined to assess the quality of LS results,

$$
\chi^{2}=\frac{(Y-<Y>) V_{\exp }^{-1}(Y-<Y>)^{T}}{N-N_{0}},
$$

where $N$ indicates the dimension of covariance of experimental data, and $N_{0}$ is the number of parameters in CTOM. The LS outputs are finally adopted when the derived cross-section curve is almost equivalent to the CTOM calculation. In this case, $\chi^{2}$ equals to 0.98 , which is close to 1.0 and indicates that the present LS results remains in good agreement with the experimental data within the uncertainty band, as shown in Figure 7. 


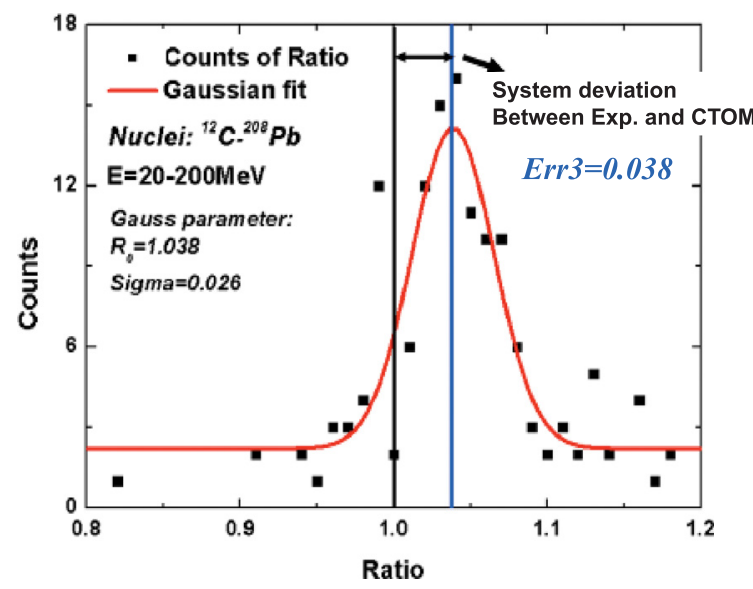

Fig. 6. The Gaussian analysis to the counts of ratio in 20 $200 \mathrm{MeV}$.

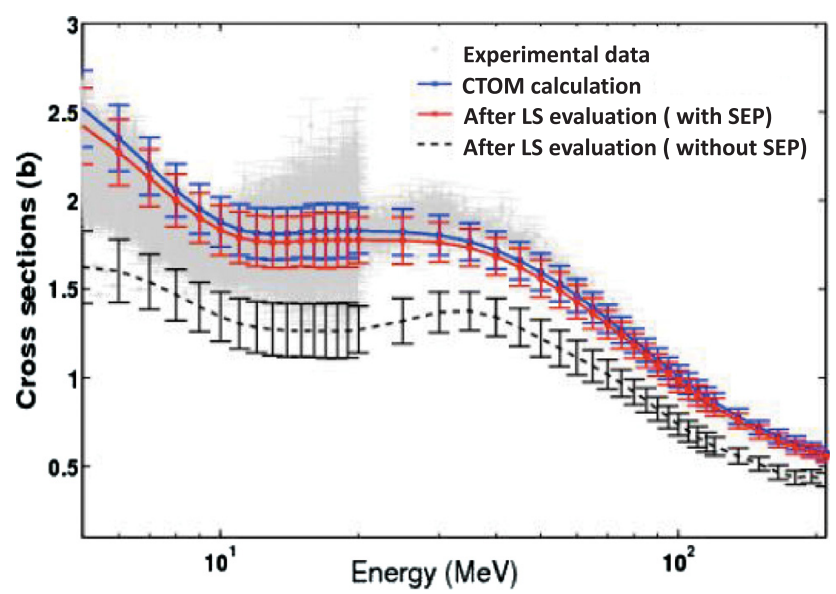

Fig. 7. The comparison of CTOM calculation, the derived cross sections with and without SEP approach in LS, and the experimental data of ${ }^{27} \mathrm{Al}(\mathrm{n}$, tot $)$.

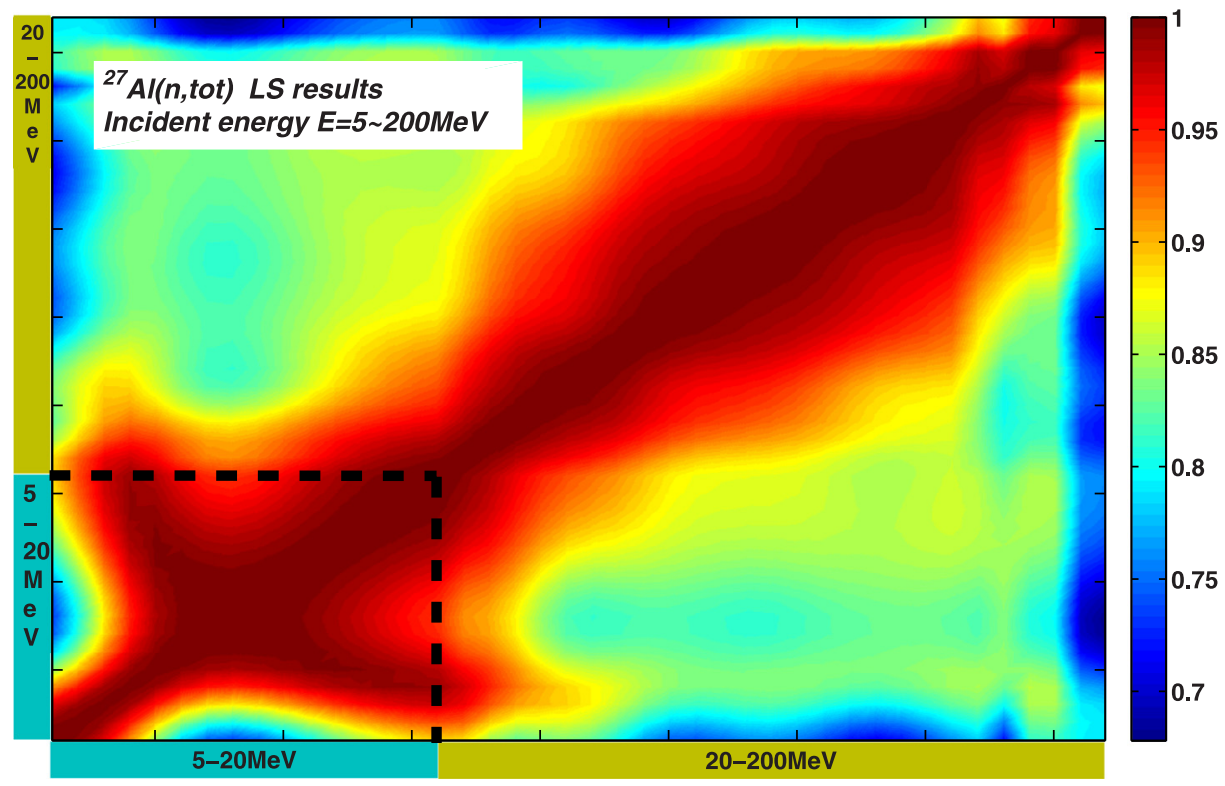

Fig. 8. The correlation matrix of the ${ }^{27} \mathrm{Al}(\mathrm{n}$, tot $)$ cross sections.

In addition, the parameters covariance matrix $\left(\mathrm{V}_{\hat{C}}\right)$ is derived simultaneously with other outputs in LS, and the covariance of CTOM predictions of any nuclide scares of measurements can also be produced in our approach.

\section{Conclusion}

In this work, the LS approach is applied to evaluate covariance data related to the (n,tot) cross sections by the nucleon-nucleus microscopic optical model CTOM. According to its application scope, the current covariance evaluation contains the analysis to ${ }^{12} \mathrm{C}-{ }^{208} \mathrm{~Pb}$ in the range $5-200 \mathrm{MeV}$. Considering the features of CTOM, the covariance is obtained through combining the information from theoretical predictions and experimental data by LS. Moreover, through this covariance analysis, the CTOM is further been proved to be powerful in the nucleon-nucleus scattering calculation, and the current scheme can be used to generate the covariance of (n,tot) cross sections predicted by CTOM for any nuclei of interest.

This work has been supported by the National Natural Science Foundation of China (Grant Nos. U1630143); We thank Prof. Z.Y. Ma, Prof. H. Muether and Prof. Q.B. Shen for their kindly discussions on CTOM calculation and covariance evaluation.

\section{Author contribution statement}

In this work, the covariance evaluation scheme was derived by Ruirui Xu, Tingjin Liu and Zhigang Ge, and the entire calculation and analysis was performed by Ruirui Xu and Zhi Zhang. The experimental data of total neutron cross sections were collected comprehensively and pre-evaluated by Zhang Yue and Xi Tao. 


\section{References}

1. G. Palmiotti, M. Assawaroongruengchot, M. Salvatores et al., J. Korean Phys. Soc. 59, 1264 (2011)

2. R. Xu, Z. Ma, E.N.E. Van Dalen et al., Phys. Rev. C 59, $034613(2012)$
3. R. Xu, Z. Ma, Y. Zhang et al., Phys. Rev. C 94, 034606 (2016)

4. R. Xu, Z. Ma, H. Muether et al., EPJ Web Conf. 146, 12009 (2017)

5. D.L. Smith, Am. Nucl. Soc., LaGrange Park, IL, USA (1991)

6. D.L. Smith et al., ANL/NDM-128 (1993)

7. A.D. Carlson, V.G. Pronyaev, D.L. Smith et al., Nucl. Data Sheets 110, 3215 (2009)

Cite this article as: Ruirui Xu, Zhigang Ge, Tingjin Liu, Yue Zhang, Xi Tao, Zhi Zhang, Covariance analysis for total neutron cross sections based on a microscopic optical model potential, EPJ Nuclear Sci. Technol. 4, 37 (2018) 